IMPACT OF COMPUTING IN SCIENCE AND ENGINEERING 4, 80-96 (1992)

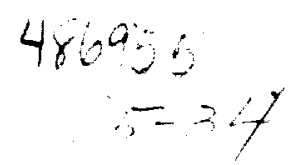

\title{
Passive Control of Supersonic Asymmetric Vortical Flows around Cones
}

N $93-1.9 \%$

\author{
C. H. LIU \\ Theoretical Flow Physics Branch, NASA Langley Research Center, \\ Hampton, Virginia 23665-5225 \\ AND \\ OSAMa A. Kandil aND Tin-CheE WONG \\ Department of Mechanical Engineering and Mechanics, Old Dominion University, \\ Norfolk, Virginia 23529-0247
}

Received November 27, 1990

\begin{abstract}
C. H. Liu, Osama A. Kandil, and Tin-Chee Wong, Passive Control of Supersonic Asymetric Vortical Flows around Cones. IMPACT of Computing in Science and Engineering 4, 80-96 (1992).

The unsteady, compressible, thin-layer Navier-Stokes equations are used to numerically study the passive control of steady and unsteady supersonic asymmetric flows around circular and noncircular cones. The main computational scheme of the present study is an implicit upwind, flux-difference splitting, finite-volume scheme. Passive control of flow asymmetry is studied by using a vertical fin in the leeward plane of geometric symmetry and side strakes with and without thickness at different orientations. The study focuses on circular-section cones since they are the most likely section-shapes for strong flow asymmetry. Side-strake passive control is shown to be more efficient and practical than vertical-fin passive control. 1992 Academic Press, Inc.
\end{abstract}

\section{INTRODUCTION}

In the moderate to high angle-of-attack (AOA) ranges, which are typical flight conditions for highly maneuverable fighter aircraft and missiles, extensive regions of vortex-dominated flow develop on the vehicle.

Within these AOA ranges, the cross-flow velocity components and the gradients of other flow variables become of the same order of magnitude as or higher than those of the axial direction. Consequently, flow separation occurs and vortices emanate from the three-dimensional separation lines of boundarylayer flows on wings, strakes, and fuselage of the vehicle. If the vortices are symmetric and stable, their influence can be exploited favorably to provide high lift and maneuverability for the vehicle. On the other hand, if the vortices 
become asymmetric or if vortex breakdown occurs, the useful influence of the vortices is terminated. Large side forces, asymmetric lifting forces, and corresponding yawing, rolling, and pitching moments, which may be larger than those provided by the vehicle control system, develop and jeopardize flight safety. The onset of buffeting due to vortex breakdown is another unfavorable vortex-induced phenomenon.

Highly swept, round- and sharp-leading-edge wings, and pointed slender bodies are common aerodynamic components of fighter aircraft and missiles. The study of vortex-dominated flow around these isolated aerodynamic components adds to our basic understanding of vortex-dominated flows. The solution of asymmetric vortex flow about slender bodies in the high AOA range is vital to the dynamic stability and controllability of fighter aircraft and missiles. The onset of flow asymmetry occurs when the relative incidence (ratio of angle of attack to nose semiapex angle) of pointed forebodies exceeds certain critical values. At these critical values of relative incidence, flow asymmetry develops due to natural and/or forced disturbances. The origin of natural disturbances may be a transient sideslip, an acoustic disturbance, or a similar disturbance of short duration. Forced disturbances, however, are caused by geometric perturbations due to imperfections in the nose geometric symmetry or similar disturbances of a permanent nature. In addition to the relative incidence as one of the determinable parameters for the onset of flow asymmetry, the freestream Mach number, Reynolds number, and body cross-section shape are important determinable parameters.

Several computational attempts have been made to simulate asymmetric vortical flows around slender bodies [1-7]. Kandil, Wong, and Liu [5] used the unsteady thin-layer Navier-Stokes equations along with two different implicit schemes to simulate asymmetric vortex flows around cones with different cross-section shapes. The numerical investigation was focused on a $5^{\circ}$-semiapex angle circular cone under locally conical flow assumption. The first computational scheme was an upwind, flux-difference splitting, finite-volume scheme and the second one was an implicit, central-difference, finite-volume scheme. The Mach number and Reynolds number being held constant at 1.8 and $10^{5}$, respectively, the angle of attack $(\alpha)$ was varied from $10^{\circ}$ to $30^{\circ}$. At $\alpha=10^{\circ}$, a steady symmetric solution was obtained and the results of the two schemes were in excellent agreement. At $\alpha=20^{\circ}$ and irrespective of the type or level of the disturbance, a steady asymmetric solution was obtained and the results of the two schemes were in excellent agreement. Two types of flow disturbances were used; a random round-off error or a random truncationerror disturbance, and a controlled transient sideslip disturbance with short duration. For the controlled transient sideslip disturbance the solution was unique, and for the uncontrolled random disturbance the solution was also unique with the exception of having the same asymmetry changing sides on the cone. At $\alpha=30^{\circ}$, an unsteady asymmetric solution with vortex shedding was obtained, and the vortex shedding was perfectly periodic. Next, the angle 
of attack was kept fixed at $20^{\circ}$ and the Mach number was increased from 1.8 to 3.0 in increments of 0.4 . The solutions showed that the asymmetry became weaker as the Mach number was increased. The flow recovered its symmetry when the Mach number reached 3.0. Selected solutions of steady and unsteady asymmetric flows were also presented for cones with elliptic and diamond cross-sectional areas.

In a later paper by the present authors [6], several issues related to the asymmetric flow solutions were addressed. It was shown that a unique asymmetric flow solution is obtained irrespective of the size of the minimum grid spacing at the solid boundary. The asymmetry could reverse sides due to the random nature of the disturbance. It was also shown that for the same flow conditions and same section fineness ratio, diamond-section cones with sharp edges have less flow asymmetry than elliptic-section cones. Again, it was also shown that unsteady periodic asymmetric flow with vortex shedding is predicted. In Ref. [7] by Kandil, Wong, and Liu, several unsteady, asymmetric vortex flows with periodic vortex shedding for circular and noncircular-section cones were presented and studied.

Experimental research efforts have also been made to control asymmetric flows for eliminating or attenuating the asymmetric forces and the resulting moments by using either passive-control [8-10] or active-control [11-13] methods. Passive-control methods include the use of a vertical fin on the leeward side along the plane of geometric symmetry [8], the use of fixed or movable forebody strakes [9-12], or the use of a rotatable forebody tip having variable cross section (from a circular shape at its base to an elliptic shape at its tip [10]). Active control methods primarily include the use of blowing ports with various blowing rates and directions on the forebody surface [11, 12]. Computational simulations have also been used to study the effectiveness of both passive [5] and active control methods [13].

In the present paper, we present a computational study for passive control of supersonic asymmetric vortical flows around cones using a vertical fin in the leeward plane of geometric symmetry and side strakes with and without thickness at different orientations. Although the present study has been focused on passive control of circular-section cones, a few applications have been considered for noncircular-section cones.

\section{FORMULATION}

\section{Governing Equations}

The three-dimensional, compressible, viscous flow around the body is governed by the conservative form of the dimensionless, unsteady, compressible Navier-Stokes equations. Introducing the time-independent body-conforming coordinates $\xi^{1}, \xi^{2}$, and $\xi^{3}$, and applying thin-layer approximations to the governing equations in $\xi^{2}$ and $\xi^{3}$ directions yields the transformed equations 


$$
\frac{\partial \bar{Q}}{\partial t}+\frac{\partial \bar{E}_{\mathrm{s}}}{\partial \xi^{\mathrm{s}}}-\frac{\partial\left(\bar{E}_{\mathrm{v}}\right)_{2}}{\partial \xi^{2}}-\frac{\partial\left(\bar{E}_{\mathrm{v}}\right)_{3}}{\partial \xi^{3}}=0 ; \quad s=1,2,3,
$$

where

$$
\bar{Q}=\frac{\hat{q}}{J}=\frac{1}{J}\left[\rho, \rho u_{1}, \rho u_{2}, \rho u_{3}, \rho e\right]^{\mathrm{t}}
$$

$\bar{E}_{\mathrm{s}} \equiv$ inviscid flux

$$
\begin{array}{r}
=\frac{1}{J}\left[\rho U_{\mathrm{s}}, \rho u_{1} U_{\mathrm{s}}+\partial_{1} \xi^{\mathrm{s} p}, \rho u_{2} U_{\mathrm{s}}+\partial_{2} \xi^{\mathrm{s} p} p, \rho u_{3} U_{\mathrm{s}}+\partial_{3} \xi^{\mathrm{s} p} p,(\rho e+p) U_{\mathrm{s}}\right]^{\mathrm{t}} \\
s=1,2,3
\end{array}
$$

$\left(\bar{E}_{\mathrm{v}}\right)_{2} \equiv$ viscous and heat-conduction flux in the $\xi^{2}$ direction

$$
=\frac{1}{J}\left[0, \partial_{k} \xi^{2} \tau_{k 1}, \partial_{k} \xi^{2} \tau_{k 2}, \partial_{k} \xi^{2} \tau_{k 3}, \partial_{k} \xi^{2}\left(u_{n} \tau_{k n}-q_{k}\right)\right]^{t}
$$

$\left(\bar{E}_{\mathrm{v}}\right)_{3} \equiv$ viscous and heat-conduction flux in the $\xi^{3}$ direction

$$
\begin{aligned}
& =\frac{1}{J}\left[0, \partial_{k} \xi^{3} \tau_{k 1}, \partial_{k} \xi^{3} \tau_{k 2}, \partial_{k} \xi^{3} \tau_{k 3}, \partial_{k} \xi^{3}\left(u_{n} \tau_{k n}-q_{k}\right)\right]^{\mathrm{t}} \\
U_{s} & =\partial_{k} \xi^{\mathrm{s}} u_{k} .
\end{aligned}
$$

The first of the three momentum elements of Eq. (5) is given by

$$
\partial_{k} \xi^{3} \tau_{k 1} \equiv \frac{M_{\infty} \mu}{\operatorname{Re}}\left(\psi \partial_{1} \xi^{3}+\phi \frac{\partial u_{1}}{\partial \xi^{3}}\right)
$$

where

$$
\phi=\partial_{k} \xi^{3} \partial_{k} \xi^{3}, \quad \psi=\frac{1}{3} \partial_{k} \xi^{3} \frac{\partial u_{k}}{\partial \xi^{3}}
$$

The second and third momentum elements are obtained by replacing the subscript 1, everywhere in Eq. (7), with 2 and 3, respectively. The last element of Eq. (5) is given by

$\partial_{k} \xi^{3}\left(u_{n} \tau_{k n}-q_{k}\right)$

$$
\equiv \frac{M_{\infty} \mu}{\operatorname{Re}}\left\{\psi \partial_{n} \xi^{3} u_{n}+\phi\left[\frac{1}{2} \frac{\partial}{\partial \xi^{3}}\left(u_{1}^{2}+u_{2}^{2}+u_{3}^{2}\right)+\frac{1}{(\gamma-1) \operatorname{Pr}} \frac{\partial\left(a^{2}\right)}{\partial \xi^{3}}\right]\right\}
$$

For Eq. (4), in the case of thin-layer approximations applied to the $\xi^{2}$ direction, the elements are given by equations similar to Eqs. (7)-(9) with the exception 
of replacing $\xi^{3}$ by $\xi^{2}$. This set of thin-layer Navier-Stokes equations is used only for the passive control cases using a vertical fin since the existence of the fin creates a second viscous thin layer which is perpendicular to the cone's thin layer. This viscous layer on the fin is in the $\xi^{3}$ direction. For the passive control cases using side strakes, thin-layer equations in $\xi^{2}$ direction are used since the viscous layers on the strakes are in the $\xi^{2}$ direction due to the method used to construct the grid around the strakes. The reference parameters for the dimensionless form of the equations are $L, a_{\infty}, L / a_{\infty}, \rho_{\infty}$, and $\mu_{\infty}$ for the length, velocity, time, density, and molecular viscosity, respectively. The Reynolds number is defined as $\operatorname{Re}=\rho_{\infty} V_{\infty} L / \mu_{\infty}$, and the pressure, $p$, is related to the total energy per unit mass and density by the gas equation

$$
p=(\gamma-1) \rho\left[e-\frac{1}{2}\left(u_{1}^{2}+u_{2}^{2}+u_{3}^{2}\right)\right] .
$$

The viscosity is calculated from the Sutherland law

$$
\mu=T^{3 / 2}\left(\frac{1+C}{T+C}\right), \quad C=0.4317
$$

and the Prandtl number $\operatorname{Pr}=0.72$.

In Eqs. (1)-(9), indicial notation is used for convenience. Hence, the subscripts $k$ and $n$ are summation indices, the superscript or subscript $s$ is a summation index. The range for $k, n$, and $s$ is $1-3$, and $\partial_{k} \equiv \partial / \partial x_{k}$.

\section{Boundary and Initial Conditions}

Boundary conditions are explicitly implemented. They include inflowoutflow conditions and solid-boundary conditions. At the plane of geometric symmetry, periodic conditions are used. At the far-field inflow boundaries, freestream conditions are specified and the outer shock is captured as part of the solution. At the far-field outflow boundaries first-order extrapolation from the interior points is used. On the solid boundary, the no-slip and no-penetration conditions are enforced $\left(u_{1}=u_{2}=u_{3}=0\right)$ and the normal pressure gradient is set equal to zero. For the temperature, the adiabatic boundary condition is enforced on the solid boundary. The initial conditions correspond to uniform flow with $u_{1}=u_{2}=u_{3}=0$ on the solid boundary.

For the passive control applications, solid-boundary conditions are enforced on both sides of the fin or the strakes.

\section{Highlights OF THE COMPUTATIONAL SCHEME}

The main computational scheme used to solve the governing equations is an implicit, upwind, flux-difference splitting, finite-volume scheme. It employs 
the flux-difference splitting scheme of Roe, which is based on the solution of the approximate Riemann problem. The inviscid flux difference at the cell interface is split into left and right flux differences according to the signs of the eigenvalues of the Roe averaged-Jacobian matrices of the inviscid fluxes $A_{s}=\partial \bar{E}_{s} / \partial \hat{q} ; s=1-3$. Flux limiters are used to eliminate oscillations in the shock region. The viscous and heat-flux terms are centrally differenced. The resulting difference equation is solved using approximate factorization in the $\xi^{1}, \xi^{2}$, and $\xi^{3}$ directions. The computational scheme is coded in the computer program "CFL3D." In this program, an implicit, flux-vector splitting, finitevolume scheme, which is based on the Van Leer scheme [14], can also be used instead of the flux-difference splitting scheme. The flux-vector splitting scheme is also used to solve for the unsteady asymmetric flow application in this paper. This application is a validation of the solution obtained previously [5] for the same application using the flux-difference splitting scheme.

Since the applications in this paper cover conical flows only, the threedimensional scheme is used to solve for locally conical flows. This is achieved by forcing the conserved components of the flow vector field to be equal at the two axial planes located at $x_{1}=0.95$ and 1.0 of the conical grid.

\section{COMPUTATIONAL STUDIES}

\section{Passive Control For a Circular Cone Using a Vertical Fin, $\alpha=20^{\circ}$}

In this section, we consider the control of steady asymmetric flow around a $5^{\circ}$-semiapex angle circular cone at an angle of attack $\alpha=20^{\circ}$, freestream Mach number $M_{\infty}=1.8$, and freestream Reynolds number $\operatorname{Re}=10^{5}$. Two vertical fins of heights $h=0.5 r$ and $r$ are placed in the leeward plane of geometric symmetry, where $r$ is the cone local radius. Figure 1 shows the results of this study, which include the total-pressure-loss contours, the surfacepressure coefficient versus the angle $\theta(\theta$ is measured from the leeward plane of geometric symmetry in the clockwise direction), and the lift coefficient versus the number of iterations. The figure also shows the results of the asymmetric flow without a vertical fin [5]. With $h=0.5 r$, the flow is still strongly asymmetric after 34,000 iterations. Comparing the case of no fin with the case of $h=0.5 r$, it is seen that the asymmetry changed sides. This is due to the random nature of the disturbance, which is a machine round-off error. With $h=0.5 r$, two vortex cores, which are connected to each other and to the body through free-shear layers, develop from the left side of the body. From the right side of the body, a free-shear layer develops and crosses over the fin to the left side of the body. It produces two vortex cores; one at each corner of the body-fin juncture with secondary separations below them. This case has been solved accurately in time but it does not show any vortex shed- 

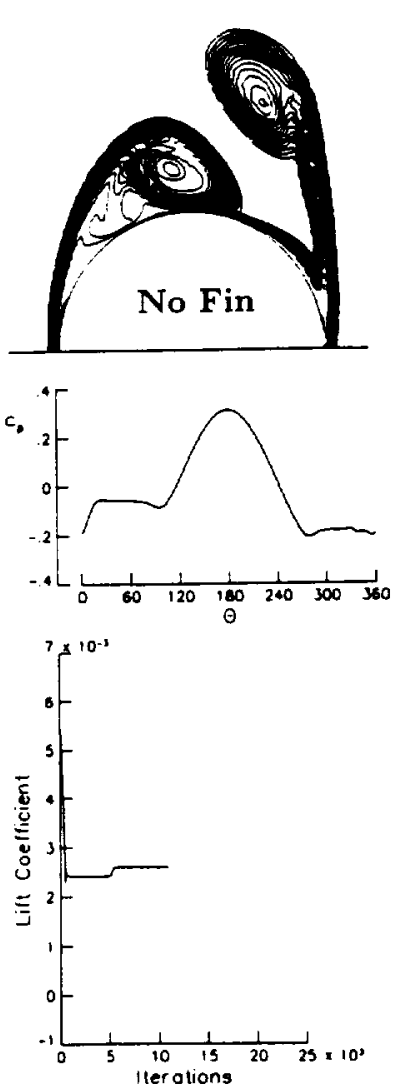
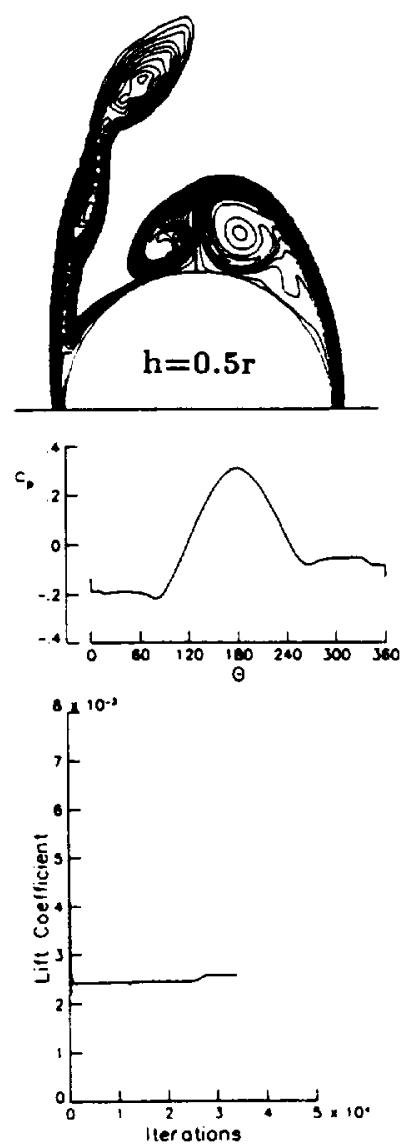
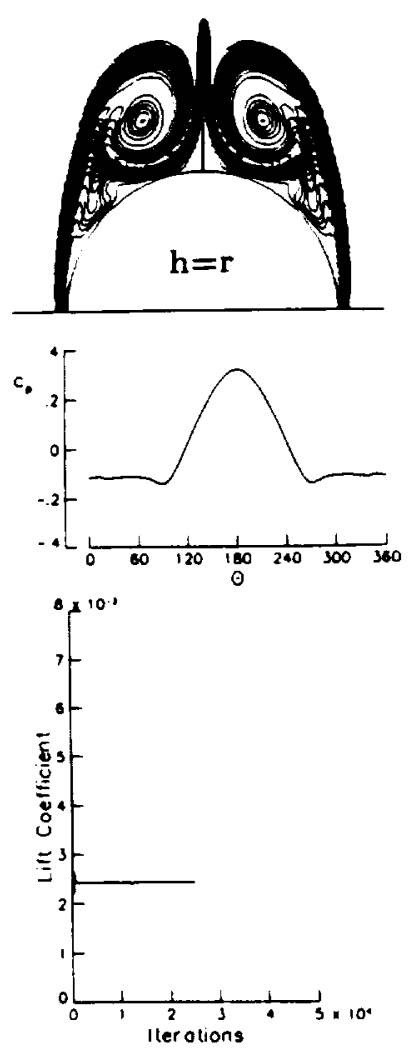

FIG. 1. Passive control of asymmetric flow around a circular cone using a vertical fin. $\alpha=20^{\circ}$, $M_{\infty}=1.8, \mathrm{Re}=10^{5}, h=0.5 r$ and $r(r=$ cone local radius $)$.

ding or unsteadiness. When the fin height is increased to $h=r$, perfect flow symmetry is obtained. The lift-coefficient curves show that when flow asymmetry develops, the lift coefficient increases over a small number of iterations and remains constant thereafter. When the flow becomes symmetric, as with $h=r$, the lift coefficient does not increase. The reason behind the flow asymmetry with $h=0.5 r$ is that the free-shear layer from the right-hand side of the body is still higher than the fin height, which allows the flow disturbances from the right and left side to interact.

Figure 2 shows a typical grid for studying passive control using a vertical fin. It contains $161 \times 81$ grid points in the wrap-around and normal directions, respectively. The grid is generated by using a modified Joukowski transformation with clustering in the normal direction at the cone surface, and clustering in the wrap-around direction at the vertical fin surfaces. The maximum radius of the computational domain is $21 r$. The figure also shows blow-ups of the cross-flow velocity in the cone-fin-juncture region for $h=0.5 r$ and $r$. It is noticed that two small recirculating bubbles exist under the vortex cores. 

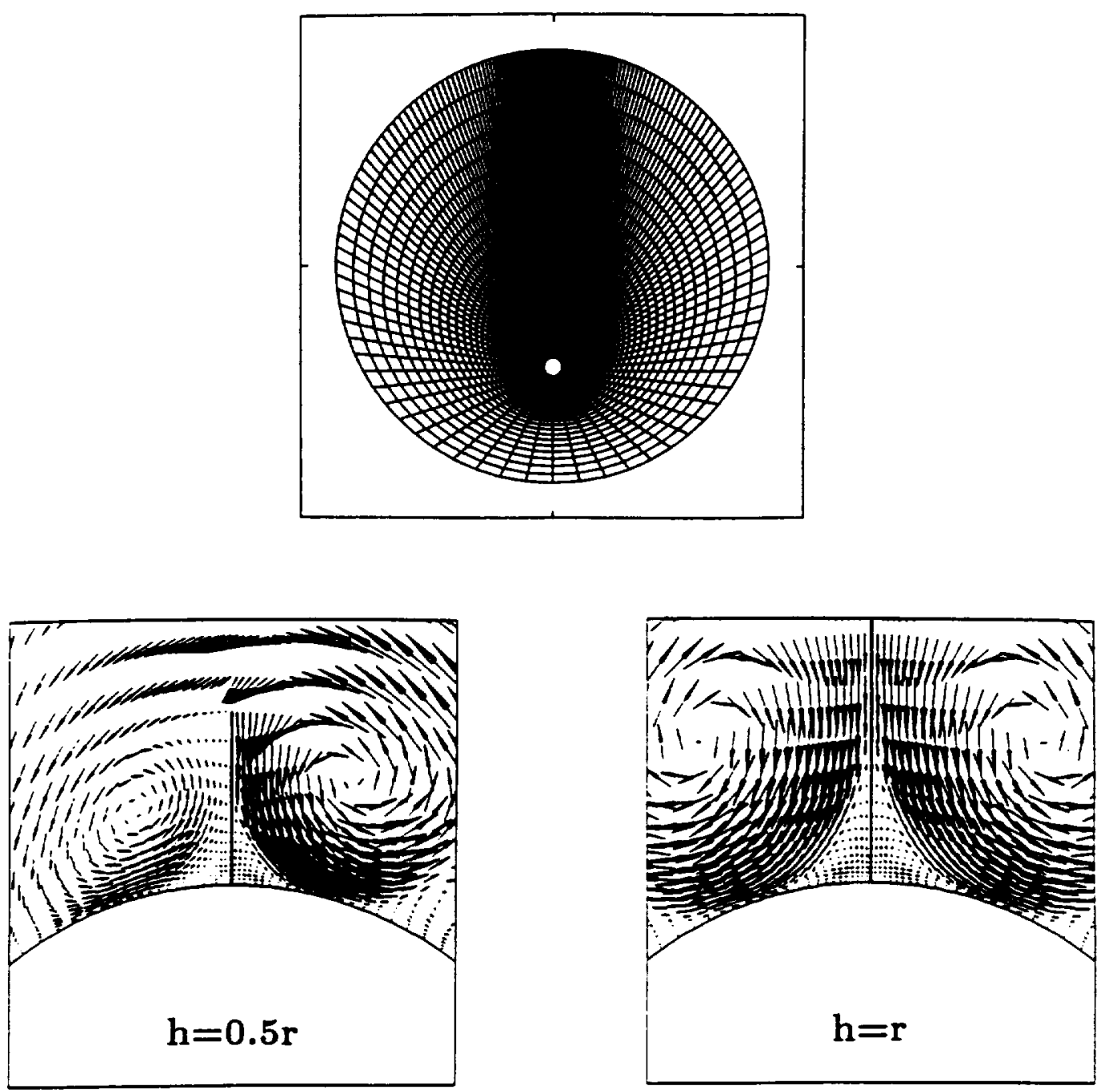

FIG. 2. A typical grid for passive control using a vertical fin $\left(161 \times 81\right.$ grid points, $\Delta \xi^{2}=\Delta \xi^{3}$ $\left.=10^{-4}\right)$ and blow-ups of cross-flow velocity in the cone-fin juncture $\left(\alpha=20^{\circ}, M_{\infty}=1.8, \operatorname{Re}=\right.$ $\left.10^{5}\right)$.

\section{Passive Control For a Circular Cone Using Side Strakes, $\alpha=20^{\circ}$}

In Fig. 3, control of flow asymmetry for the same flow conditions as in the first application is considered using sharp-edged thick strakes of height $h=$ $0.3 r$. The side-strakes render the flow perfectly symmetric since the two primary vortex cores, left and right, are pushed further apart preventing the flow disturbances of the two sides from interacting. It is easily seen that there are four vortices on each side; one is a primary vortex and three are secondary vortices. The pressure-coefficient curve shows jumps at $\theta=90^{\circ}$ and $270^{\circ}$, where the strakes are located. These jumps change the shape of the $C_{\mathrm{p}}$-curve in comparison with that of the vertical-fin-control case. Moreover, the lift coefficient of the side-strake-control case is double that of the vertical-fin-control case. 

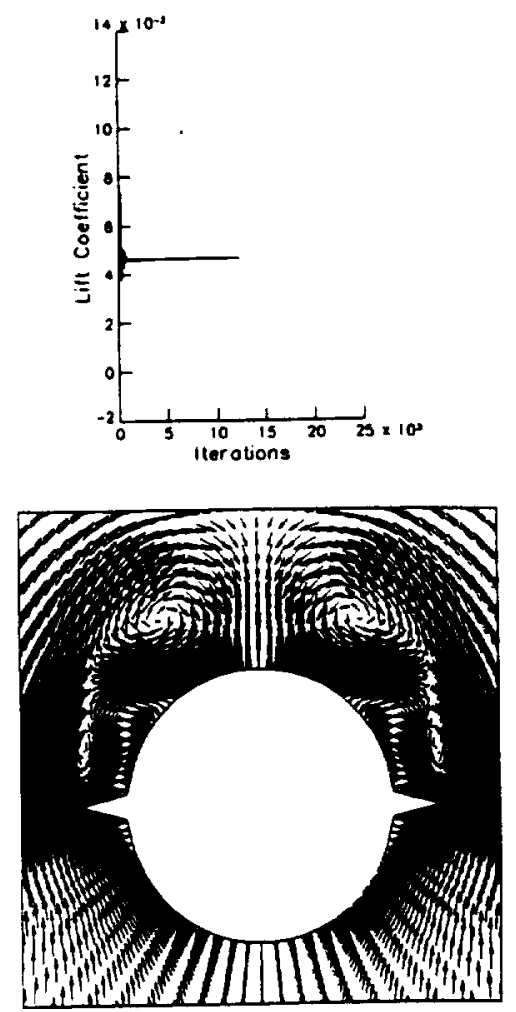
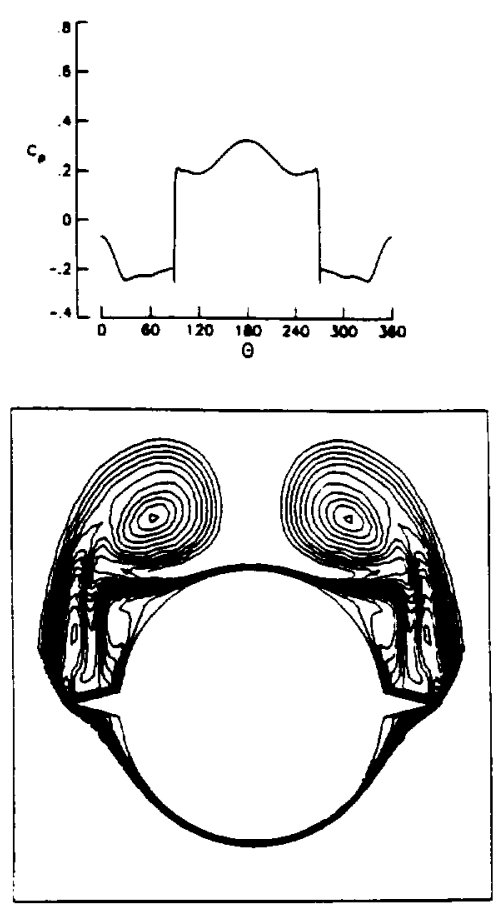

Fig. 3. Passive control of asymmetric flow around a circular cone using sharp-edged thick strakes. $\alpha=20^{\circ}, M_{\infty}=1.8, \operatorname{Re}=10^{5}, h=0.3 r$.

It is concluded that side-strake control in comparison with vertical-fin control not only is efficient in providing higher lift, but also is more practical due to the strake's shorter height, and hence lesser weight.

\section{Passive Control For a Circular Cone Using Side Strakes with and without Thickness, $\alpha=30^{\circ}$}

In this case, the cone angle of attack is increased to $30^{\circ}$ keeping all the other flow conditions fixed. This flow application has been solved previously in Ref. [5] by Kandil, Wong, and Liu using a flux-difference splitting (FDS) scheme. The results showed unsteady asymmetric flow with periodic vortex shedding. The total-pressure-loss contours of the time steps from 15,000 to 15,700 , representing one-half the cycle during the periodic response, is shown in Fig. 4. Other unsteady asymmetric flows with periodic vortex shedding around elliptic-section and diamond-section cones have also been presented by the authors in Refs. [5, 7]. In all these applications, the FDS scheme was used. In order to show that the unsteady asymmetric solutions are not scheme dependent, the effect of computational methodologies and numerical dissipation on the solutions are examined in the present paper. The case of asymmetric flow around a circular cone has also been computed using the flux- 

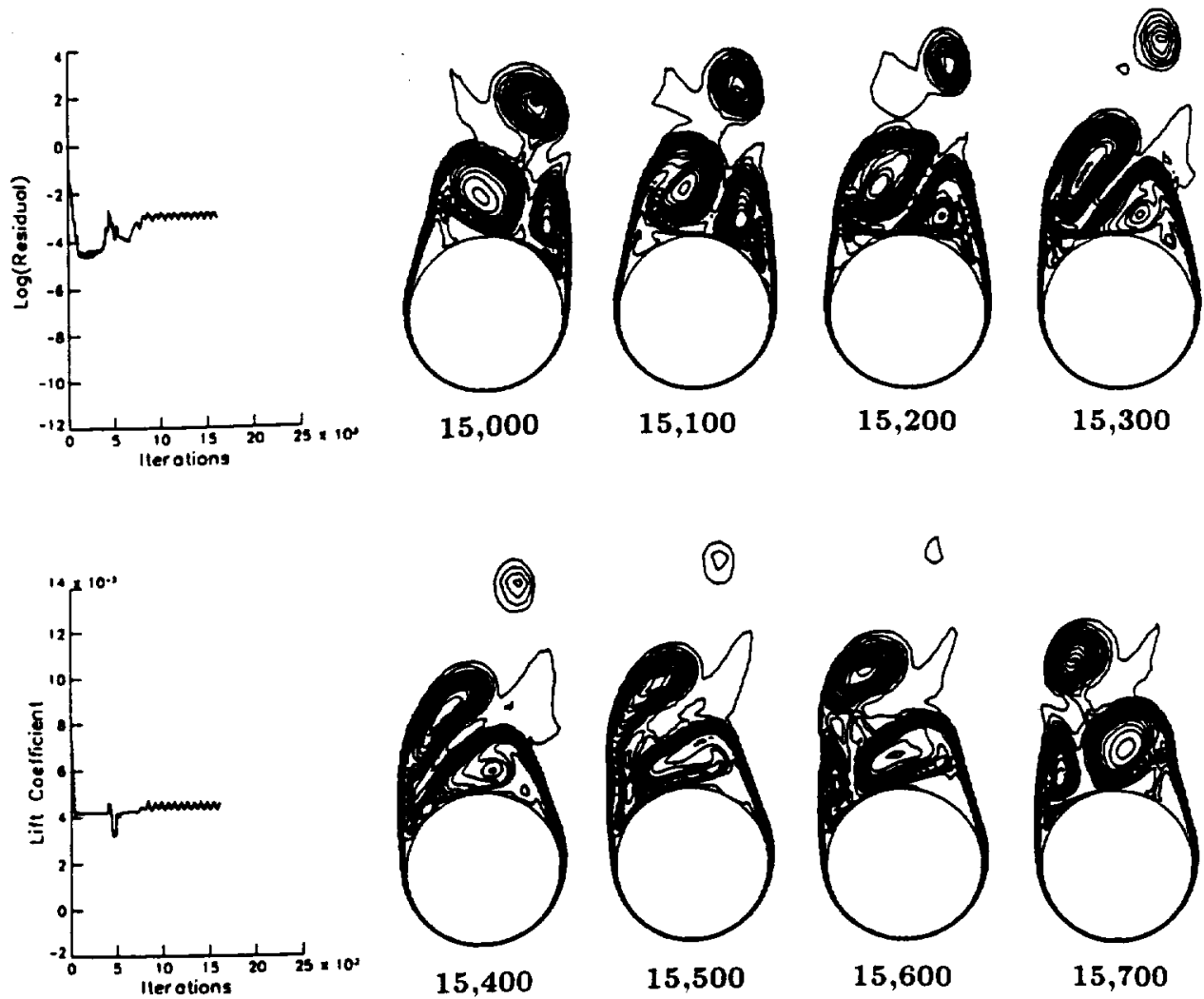

Fig. 4. Unsteady asymmetric flow with vortex shedding around a circular cone using FDS schemes. $\alpha=30^{\circ}, M_{\infty}=1.8, \operatorname{Re}=10^{5}, \Delta t=10^{-3}$.

vector splitting (FVS) scheme of the same code. In Fig. 5, we show the timeaccurate solutions using the FVS scheme on the same grid. Using the FVS scheme, the flux limiters are turned on, and as can be seen from the logarithmic-residual curve, the solution becomes symmetric and steady after 5000 time steps. Next, the flux limiters are turned off, and the solution shows a transient response up to 12,000 time steps. Thereafter, the solution becomes periodic with periodic asymmetric vortex shedding. The solution is shown every 100 time steps starting from time step 13,900 until time step 14,600. Although the process of adjusting the time instants to match those of the FDS solution is difficult, it is seen that the captured snap shots of the FVS solution almost match those of the FDS solution at time steps $15,000,15,100,15,200$, $15,300,15,400,15,500,15,600$, and 15,700 , respectively. Comparing the FVS solutions at time step of 13,900 with that of 14,600 , it is seen that they are mirror images of each other. Hence, periodic flow response has been achieved with a period of $1,400 \times 10^{-3}=1.4$, which is exactly the same period of shedding as that of the FDS solution. This pinpoints the high numerical dissipation effect of the FVS scheme when the flux limiters are turned on. The resulting numerical dissipation in the FVS is large enough to dampen the random disturbances of the flow solution. By turning off the flux limiters in 


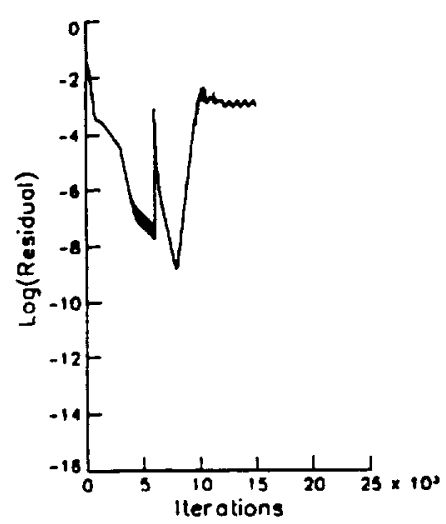

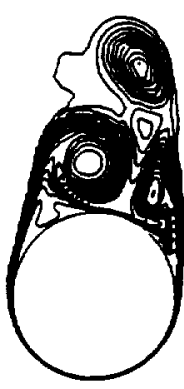

13,900

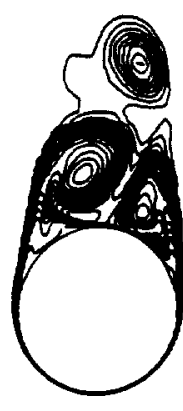

14,000

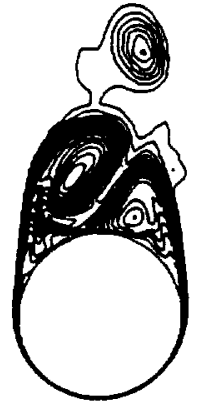

14,100

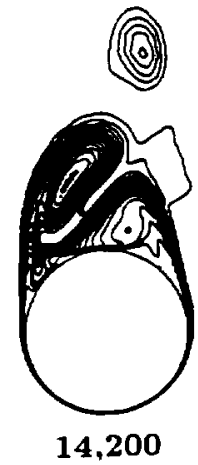

(O)

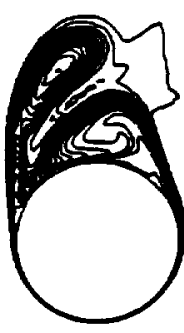

14,300
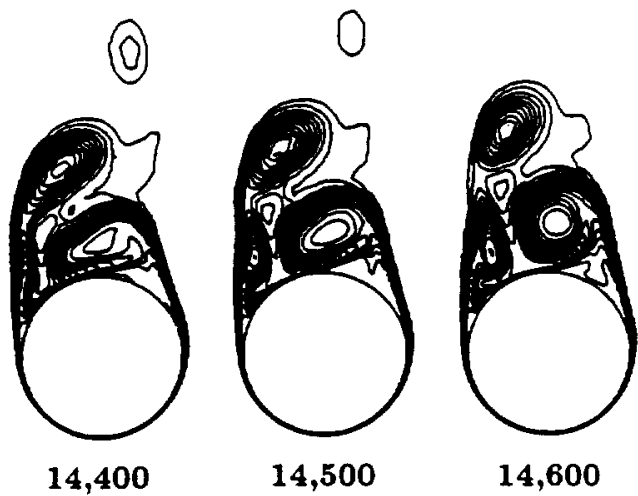

FIG. 5. Unsteady asymmetric flow with vortex shedding around a circular cone using FVS schemes. $\alpha=30^{\circ}, M_{\infty}=1.8, \operatorname{Re}=10^{5}, \Delta t=10^{-3}$.

the FVS scheme, the random disturbances can grow producing the asymmetric unsteady vortex shedding. This also shows that the FDS scheme, even with the flux limiters turned on, is less dissipative than the FVS scheme. These results conclusively show that unsteady asymmetric flows are obtained irrespective of the numerical methodologies.

Next, we consider the control of this unsteady asymmetric flow using sharpedged thick strakes and flat-plate strakes with different orientations. For all the strake shapes, the height is kept at $0.3 r$. Figure 6 shows the results of this study using sharp-edged and flat-plate strakes at $\delta=0^{\circ}, 10^{\circ}$, and $-10^{\circ}$, where $\delta$ is the angle measured in the counter-clockwise direction from the horizontal line at $\theta=90^{\circ}$. For this angle of attack, all the strake orientations are still effective in eliminating the unsteady asymmetric vortex shedding and rendering the flow perfectly symmetric. Again the $C_{p}$-curves show jumps at the strakes leading edges at $\theta=90^{\circ}$ and $270^{\circ}$. The lift coefficient of all controlled flow cases, Fig. 7 , is higher than that of the asymmetric flow case. With slight differences in the lift coefficient, the highest lift is produced by the flat-plate strakes with $\delta=-10^{\circ}$, where the primary vortex cores are slightly closer to the body surface than for the other cases. 

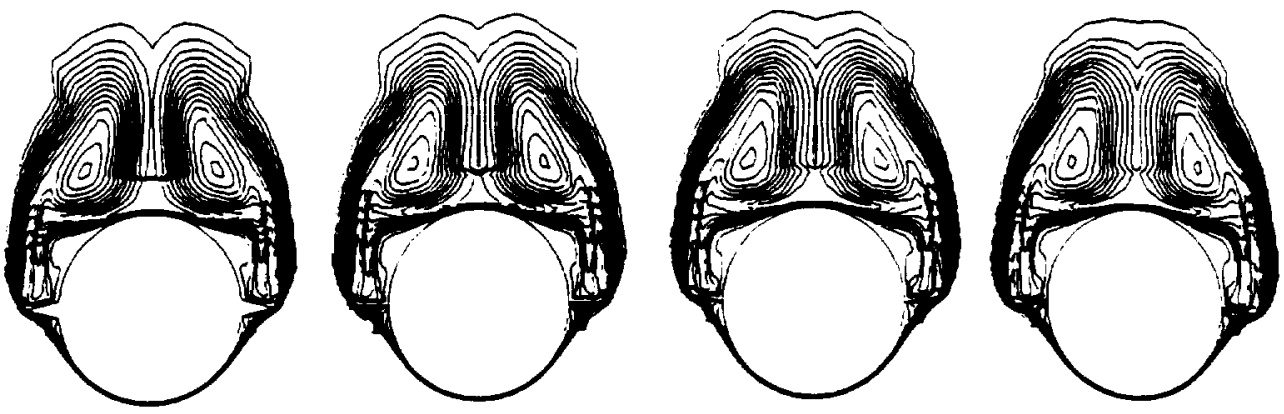

$\delta=0^{\circ}$

$\delta=10^{\circ}$

$\delta=-10^{\circ}$
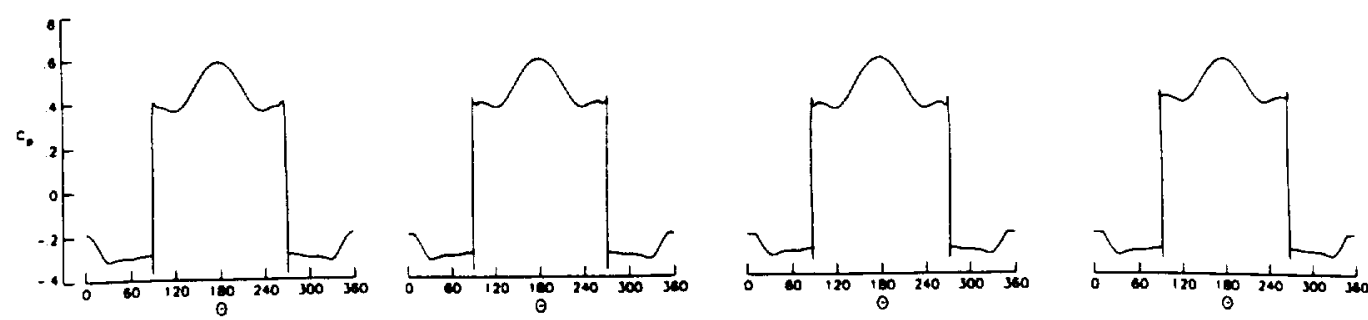

FIG. 6. Passive control of asymmetric flow around a circular cone using sharp-edged thick strakes and flat-plate strakes with different orientations. $\alpha=30^{\circ}, M_{\infty}=1.8, \operatorname{Re}=10^{5}, h=0.3 r$.
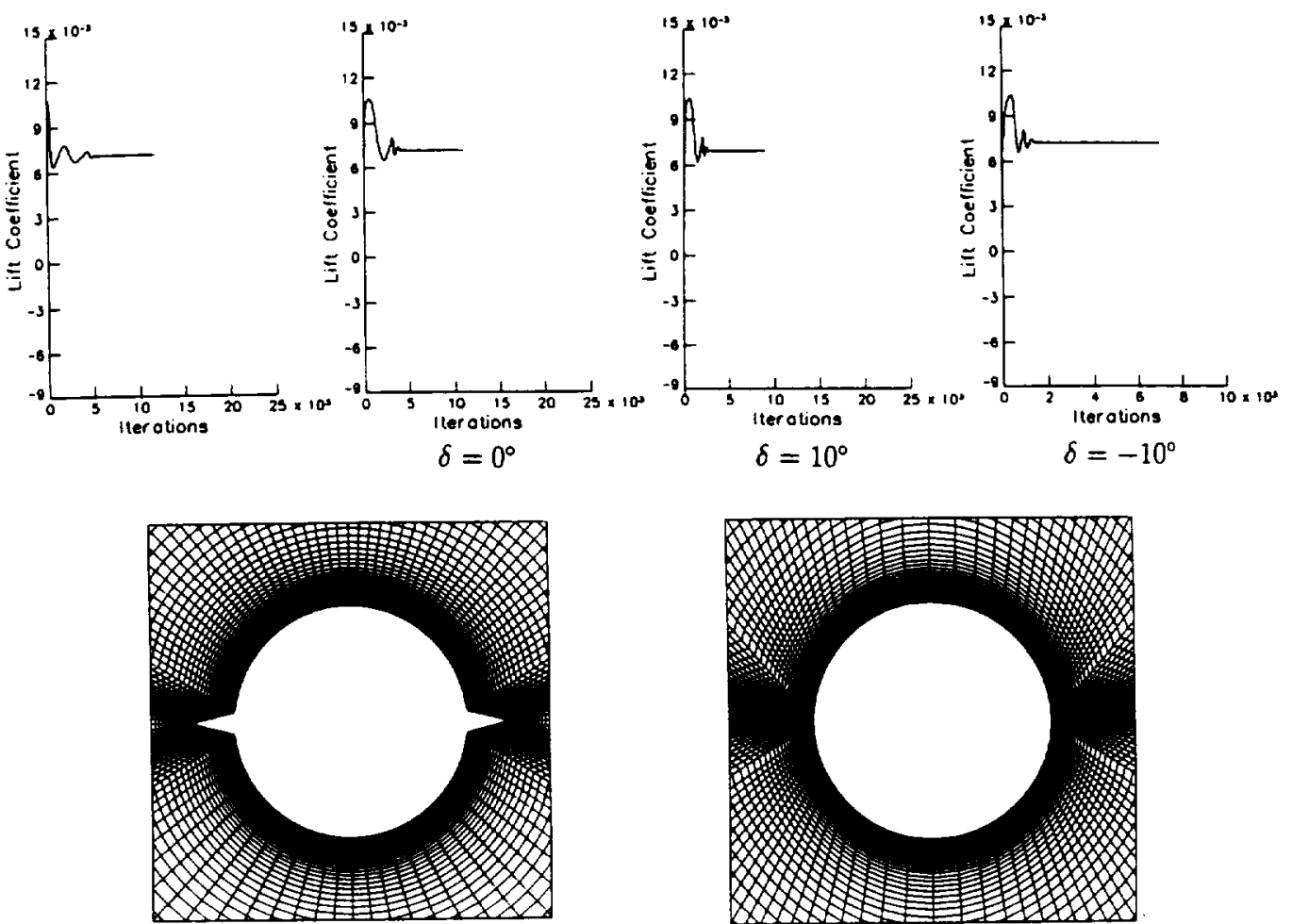

$\delta=10^{\circ}$

FIG. 7. Lift coefficients and typical grids for passive control using side strakes. $\alpha=30^{\circ}, M_{\infty}=$ $1.8, \operatorname{Re}=10^{5}, h=0.3 r$. 
Comparing the results of the sharp-edged thick strakes at $\alpha=30^{\circ}$ with those of the sharp-edged thick strakes at $\alpha=20^{\circ}$ (Fig. 3), we notice that the primary vortex cores of the former are closer to the plane of symmetry and higher above the cone surface than those of the latter.

In the bottom row of Fig. 7, we show typical grids for the cases of sharpedged thick strakes and the flat-plate strakes with $\delta=10^{\circ}$. The grids are generated by using a hyperbolic grid generator with transfinite grid interpolation to refine the grid in the strake region.

4. Passive Control For a Circular Cone Using Sharp-Edged Thick Strakes,

$$
\alpha=40^{\circ}
$$

For this case, the angle of attack is increased to $\alpha=40^{\circ}$ keeping all the other flow conditions fixed. The same sharp-edged thick strakes of the previous case $\left(\alpha=30^{\circ}\right)$ have been used along with the same grid. Figure 8 shows the results of this case. It is seen that although the $C_{\mathrm{p}}$-curve looks perfectly symmetric and although the lift coefficient curve does not show any increase after the time step 4,000, the total-pressure-loss contours show very slight asymmetry. This indicates that the current height of the strakes might not be suf-
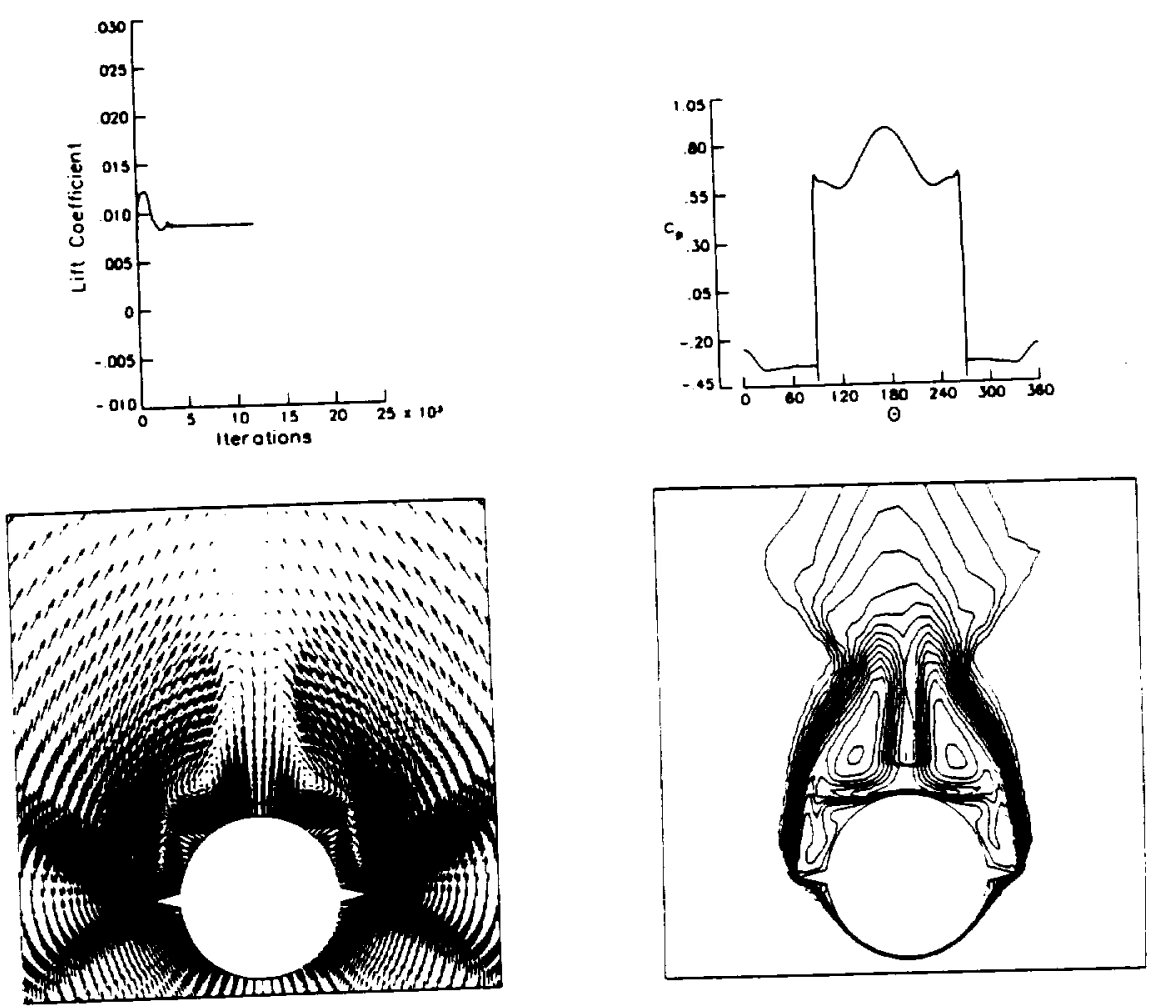

FIG. 8. Passive control of asymmetric flow around a circular cone using sharp-edged thick strakes. $\alpha=40^{\circ}, M_{\infty}=1.8, \operatorname{Re}=10^{5}, h=0.3 r$. 
ficient to yield flow symmetry at higher angles of attack. The present solution shows that the vortical flow substantially stretches upwards.

\section{Passive Control For an Elliptic-Section Cone Using a Vertical Fin, $\alpha=25^{\circ}$}

In order to produce a substantial flow asymmetry (of the same order as that of the circular cone of Fig. 1) for an elliptic-section cone of fineness ratio fr $=0.6$, the angle of attack has been increased to $\alpha=25^{\circ}$, and the freestream Mach number has been decreased to $M_{\infty}=1.5$. Passive control of this flow has been tested using vertical fins of heights $h=1.5 a$ and $2 a$, where $2 a$ is the length of the cross-section minor axis. The grid is $161 \times 81$ in the wraparound and normal directions, and was generated by a modified Joukowski transformation with minimum grid spacing of $\Delta \xi^{2}=10^{-4}$ and $\Delta \xi^{3}=10^{-4}$ (for the vertical-fin control). The results are shown in Figs. 9 and 10. For the case with no fin, the lift coefficient shows an increase near step 7,000, and it remains constant thereafter. For the case with $h=1.5 a$, the flow is still strongly asymmetric and the lift coefficient shows an increase near step 8000 . The total-pressure-loss contours show a very long free-shear layer from the left side. From the right side, the free-shear layer becomes higher than the vertical fin and crosses over the fin to the left side. Two primary-vortex cores are

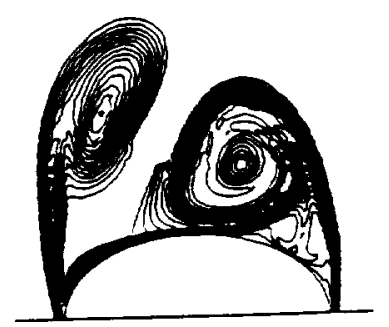

No Fin

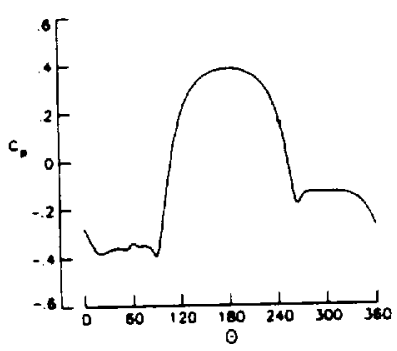

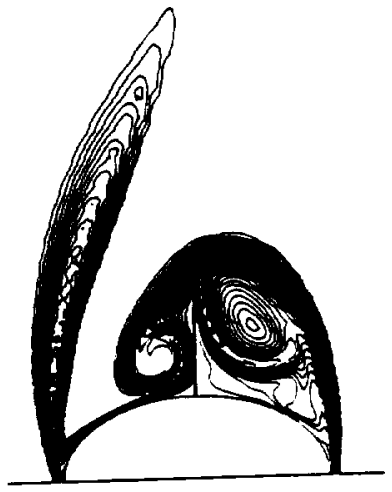

$h=1.5 a$

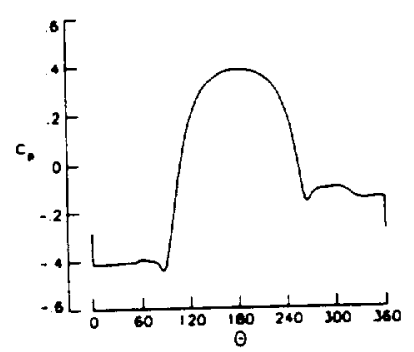

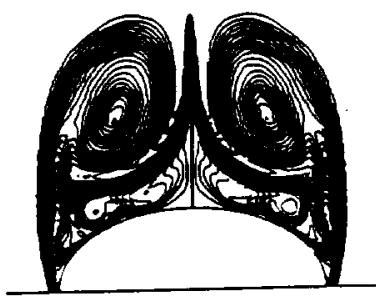

$\mathrm{h}=\mathbf{2 a}$

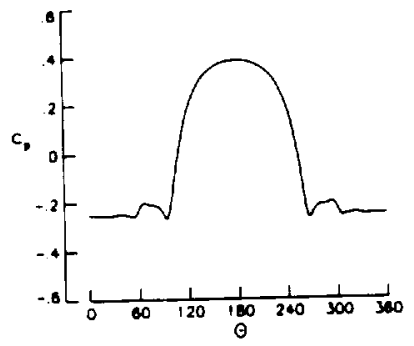

FIG. 9. Passive control of asymmetric flow around an elliptic-section cone using vertical fin. $\alpha$ $=25^{\circ}, M_{\infty}=1.5, \operatorname{Re}=10^{5}, \mathrm{fr}=0.6, h=1.5 a$ and $2 a$ ( $2 a=$ cone local minor-axis length). 


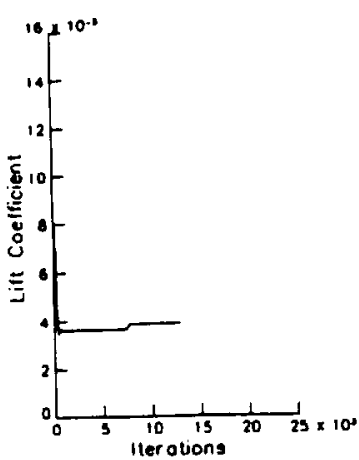

No Fin

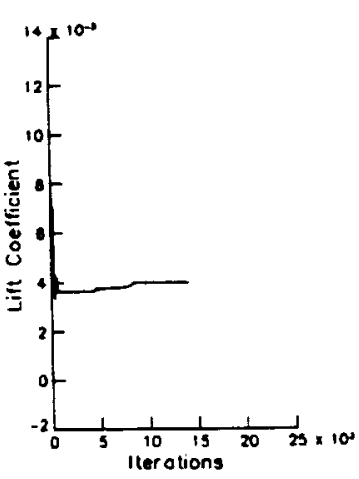

$\mathrm{h}=1.5 \mathrm{a}$

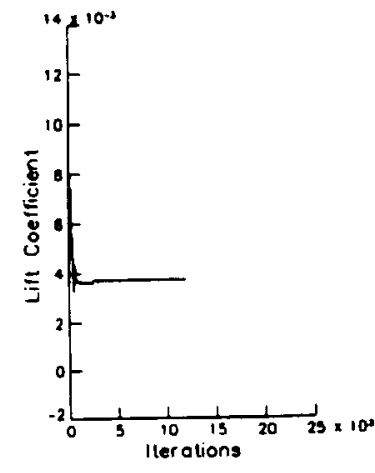

$\mathbf{h}=\mathbf{2 a}$

FIG. 10. Comparison of lift coefficients for flow over an elliptic-section cone with and without a vertical fin. $\alpha=25^{\circ}, M_{\infty}=1.5, \operatorname{Re}=10^{5}, \mathrm{fr}=0.6, h=1.5 a$ and $2 a$.

formed at the cone-fin juncture with secondary separations below them. When the fin height is increased to $h=2 a$, perfect symmetric flow is obtained and the lift coefficient remains constant. The behavior of this vertical-fin control case is very similar to that of the circular cone. As long as the vertical fin is high enough as compared to the maximum height of the free-shear layer, flow symmetry is obtained. Obviously, if side-strakes are used, they will push the vortex cores further apart preventing disturbance interaction between the two sides, and flow symmetry will be achieved.

6. Passive Control For a Diamond-Section Cone Using a Vertical Fin, $\alpha=25^{\circ}$

For this case, the section fineness ratio is 0.8 , the angle of attack is $\alpha=$ $25^{\circ}$, and the freestream Mach number is 1.5 . With the exception of the section fineness ratio, the flow conditions of this case are the same as those of the elliptic-section cone. This simply shows that for the same section fineness ratio and same flow conditions, diamond-section cones with sharp edges produce less flow-asymmetry strength than that of elliptic-section cones.

Figure 11 shows the results of the diamond-section cone flow with and without a vertical fin. For the flow-control case, a symmetric flow has been obtained using a vertical fin of height $h=1.5 a$, which is shorter than that required for the elliptic-section case.

\section{CONCLUDING REMARKS}

Computational studies for passive control of steady and unsteady, supersonic, asymmetric vortical flows have been carried out using vertical fins in the leeward plane of geometric symmetry, and using side strakes with and without thickness. The governing equations are the unsteady, compressible, thin-layer Navier-Stokes equations. The equations have been solved using 

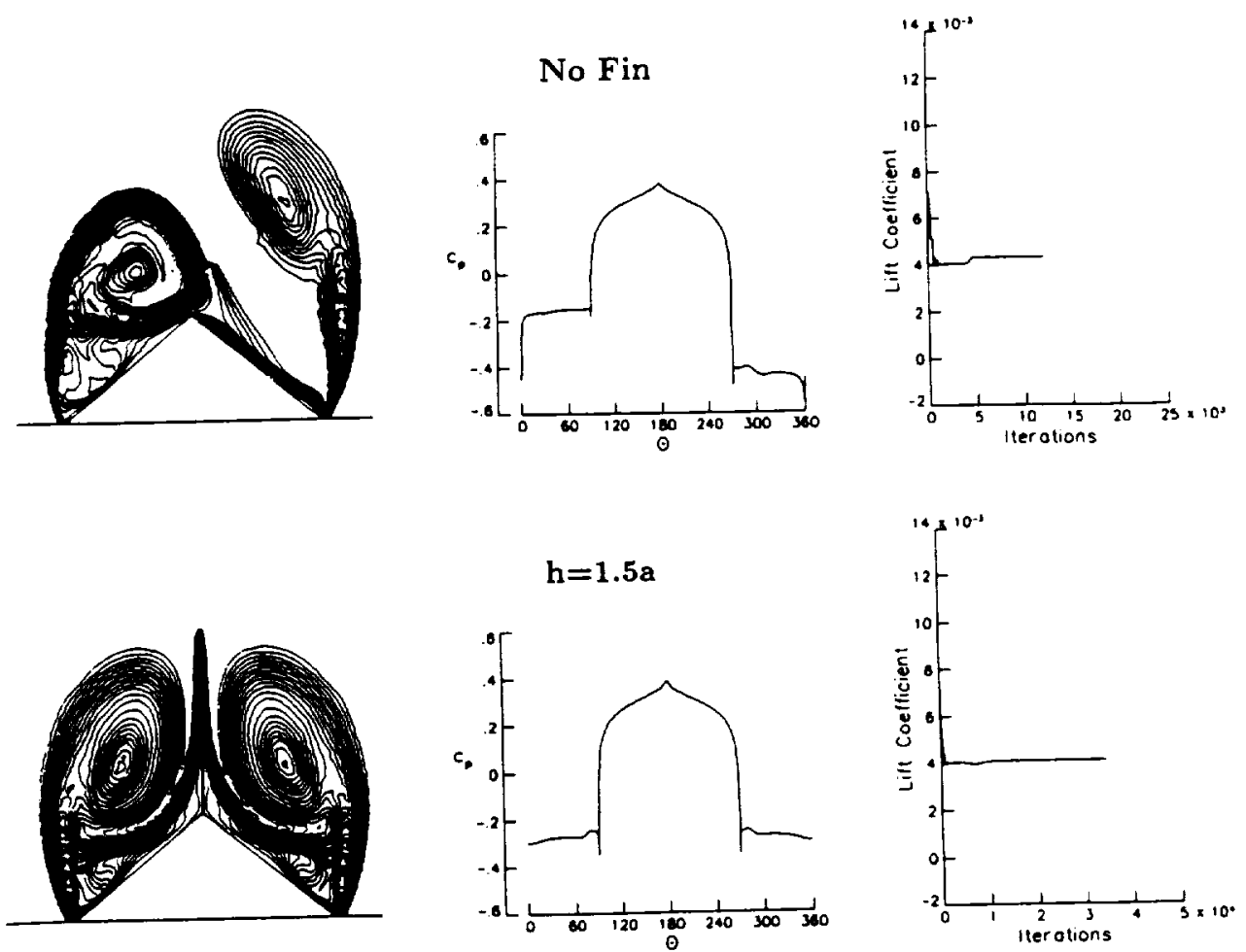

FIG. 11. Passive control of asymmetric flow around a diamond-section cone using a vertical fin. $\alpha=25^{\circ}, M_{\infty}=1.5, \operatorname{Re}=10^{5}, \mathrm{fr}=0.8, h=1.5 a$.

an implicit, upwind, flux-difference splitting, finite-volume scheme. The flowcontrol studies have focused on circular-section cones since they are the best potential section-shapes for strong flow asymmetry. It has been shown that side-strake passive control is very effective over a wide range of angle of attack. It has also been shown that side-strake control is more efficient than verticalfin control in producing higher lift. Moreover, it is more practical since the strakes have shorter height and, hence, less weight in comparison with the vertical fin.

\section{ACKNOWLEDGMENTS}

This research work has been supported by the NASA Langley Research Center under Grant NAS1-18584-71 for the last two authors.

\section{REFERENCES}

1. J. E. Graham and W. L. Hankey, Computation of the asymmetric vortex pattern for bodies of revolution. AlAA J. 23, 1500-1504 (1983).

2. D. Degani and L. B. Schiff, Numerical simulation of the effect of spatial disturbances on 
vortex asymmetry. In AIAA 28th Aerospace Sciences Meeting, Reno, Nevada. AIAA 89-0340 (1989).

3. D. Degani, Numerical investigation of the origin of vortex asymmetry. In AIAA 28th Aerospace Sciences Meeting, Reno, Nevada. AIAA 90-0593 (1990).

4. M. J. Siclari, Asymmetric separated flows at supersonic speeds. In AlAA 28th Aerospace Sciences Meeting, Reno, Nevada. AIAA 90-0595 (1990).

5. O. A. Kandil, T. C. Wong, and C. H. Liu, Prediction of steady and unsteady asymmetric flows around cones. In AIAA 28th Aerospace Sciences Meeting, Reno, Nevada. AIAA 90$0598(1990)$.

6. O. A. Kandil, T. C. Wong, and C. H. Liu, Asymmetric supersonic flow around cones with noncircular sections. AGARD Paper No. 16, in Symposium on Missile Aerodynamics, Friedrichshafen, Germany (1990).

7. O. A. Kandil, T. C. Wong, and C. H. Liu, Numerical simulation of steady and unsteady asymmetric vortical flows. ASME Symposium on Non-Steady Fluid Dynamics, Toronto, Ontario. FED-Vol. 92, pp. 99-108, (1990).

8. W. Stahl, Suppression of asymmetry of vortex flow behind a circular cone at high incidence. In AlAA Atmospheric Flight Conference, Boston, Massachusetts. AIAA 39-3372-CP, pp. 231236 (1989).

9. T. T. Ng, On leading edge vortex and its control. In AlAA Atmospheric Flight Conference, Boston, Massachusetts. AIAA 89-3346-CP, pp. 1-15 (1989).

10. C. Moskovitz, R. Hall, and F. DeJarnette, Experimental investigation of a new device to control the asymmetric flowfield on forebodies at large angles of attack. In AIAA 28th Aerospace Sciences Meeting, Reno, Nevada. AlAA 90-0069 (1990).

11. A. M. Skow and D. J. Peake, Control of the forebody vortex orientation by asymmetric air injection, Part B-Details of the flow structure. In AGARD-LS-121, High Angle-of-Attack Aerodynamics, pp. 10.1-10.22 (1982).

12. T. T. Ng, Aerodynamic control of nasp-type vehicles through vortex manipulation. In AIAA 28th Aerospace Sciences Meeting, Reno, Nevada. AIAA 90-0594 (1990).

13. D. A. Travella, L. B. Schiff, and R. M. Cummings, Pneumatic vortical flow control at high angles of attack. In AIAA 28th Aerospace Sciences Meeting, Reno, Nevada. AIAA 90-0098 (1990).

14. L. C. Rumsey and W. K. Anderson, Some numerical aspects of unsteady Navier-Stokes computations over airfoils using dynamic meshes. In AIAA 26th Aerospace Sciences Meeting. Reno, Nevada. AlAA 88-0329 (1988). 